\title{
SOBRE LA OBRA DE ROBERT M. MacIVER La acción social entre la comunidad y el Estado
}

\author{
MANUEL HERRERA GÓMEZ y PEDRO CASTÓN BOYER
}

Universidad de Granada

PALABRAS CLAVE ADICIONALES

Teoría sociológica, Epistemología de las Ciencias Sociales, Filosofía y Metodología de las Ciencias Sociales.

\section{ADDITIONAL KEYWORDS}

Sociological Theory, Epistemology of Social

Sciences, Philosophy and Methodology of Social Sciences.

RESUMEN. Sociología, ciencia y filosofía política son los ámbitos disciplinares que han guiado y alimentado, compenetrándose, la investigación de Robert M. Maclver. Todo esto es debido a una intuición bien precisa que está presente en la base de su pensamiento: estudiar la acción social para fundar una teoría realista de la democracia con importantes contenidos federales en virtud de una concepción de la relación entre sociedad y Estado en la que este último es un simple medio perteneciente a la esfera de la civilización respecto a la sociedad entendida como realidad originaria (como red de grupos y comunidades), en la que se constituyen y encuentran expresión los intereses primarios de todo ser social.

ABSTRACT. Sociology, science and political philosophy are the disciplinary fields that have guided Robert M. Maclver's investigation. This is based on accurate intuition always present in his thought: to study human social action in order to set up a realistic theory on democracy with important federal contents by reason of an understanding of the relation between society and state. At this relation, state is a simple mean belonging to the sphere of civilization and society is an original reality (a "network of groups and communities"), in which the primary interests of any social being are expressed.

E-mail: mherrera@ugr.es pcaston@ugr.es

Revista Internacional de Sociología (RIS)

Tercera Época, No 39, Septiembre-Diciembre, 2004, pp. 225-256. 
RIS

REvista INTERnacional DE Sociologla

№ 39, SEPTIEMBRE-DICIEMBRE, 2004

MANUEL HERRERA Y PEDRO CASTÓN

\section{INTRODUCCIÓN}

En As a Tale That Is Told, la autobiografia que Robert M. MacIver publica dos años antes de su muerte (1970), emergen constantemente dos temas que marcan el sentido global de la obra de este filósofo y sociólogo de origen escocés. Por una parte, MacIver recuerda cómo el problema de la "comunidad" ha constituido "el tema central de mi trabajo" y cómo "el título de mi primer libro (Comunity) fue profético"; por otra parte, reafirma el haberse sentido siempre "un liberal en el sentido literal de la palabra" (MacIver, 1968:130 y 226).

La integración de estos dos aspectos, el sociológico (que estudia la acción social en la interacción creativa que establece con las estructuras de la sociedad) y el ético (que canaliza el propio interés científico sin desnaturalizarlo), constituye las dos dimensiones de una obra en la que MacIver "revela la misma voluntad y capacidad de contemplar una visión integrada de los problemas que afronta (...) y que, entre otras cosas, resulta de la sucesión, en las designaciones de su posición académica, de ciencia política y sociología" (Poggi, 1962:8). En este sentido, la posición de MacIver, la irreductibilidad de su planteamiento "respecto a las exigencias de la usual departamentalización de las disciplinas políticas y sociales en los Estados Unidos", ha sido advertida y considerada en más de una ocasión "como un rasgo distintivo" (Poggi, 1962:9) que, incluso, le permitió huir de los fáciles conformismos científicos para elaborar unas aportaciones que todavía son originales en la sociología americana.

La sociología de MacIver es una verstehende Soziologie deudora de la sociología europea: concretamente de las aportaciones de Ferdinand Tönnies y de Alfred y Max Weber, ya que en "la profundidad y riqueza de sus formulaciones, $y$ en las investigaciones que ha impulsado, ha construido una obra fundamental en la que pueden apoyarse los futuros teóricos" (Spitz, 1964:312).

En España, Salvador Giner (1971, 1974, 1979a y 1979b) ha sido pionero en el estudio del tema de la comunidad en Tönnies y en sus contemporáneos, analizando con profundidad la problemática abierta a partir de los actores anteriormente citados en el campo de las ciencias sociales. Sobre la noción clave de "acción social" se centraron los esfuerzos de Tönnies, Weber, Pareto, Durkheim, Simmel y Freud para construir una sólida ciencia. "Esencialmente se trata de la búsqueda de un fenómeno, y su correspondiente concepto, que pueda servir de asidero para la sociología, que pueda conferirle la necesaria solidez" (Giner, 1979b: 10-11).

Sociología, ciencia y filosofía política son los ámbitos disciplinares que han guiado y alimentado, compenetrándose, la investigación de Robert M. MacIver. Ahora bien, conviene matizar que esto es debido a una intuición bien precisa que, desde sus primeras obras, está presente en la base de su pensamiento: estudiar la acción social humana para fundar una teoría realista de la democracia con importantes contenidos federales en virtud de una concepción de la relación entre sociedad y Estado en la que este último es un simple medio, un instrumento 
perteneciente, como dice MacIver (planteando la tripartición alfredweberiana de cultura, civilización y proceso social), a la esfera de la civilización respecto a la esfera de la sociedad, entendida esta última como realidad originaria, como "red de grupos y comunidades" en la que se constituyen y encuentran expresión los intereses primarios de todo ser social.

En la esfera de la sociedad el hombre se presenta como miembro de un grupo y como individuo. Estamos ante dos características fundamentales que, si son consideradas aisladamente, nos llevan a conclusiones "opuestas pero igualmente erróneas" (MacIver, 1933:24). La solución teórica de la relación individuo-sociedad que MacIver proyecta al final de sus primeras obras insiste, por una parte, en la irreductibilidad (recíproca) de Estado y sociedad y, por otra, en la posibilidad de que una adecuada praxis democrática consiga ordenar esta continua confrontación, esta dialéctica fundada en un dato incontrovertible cuyo reconocimiento es la condición misma de la libertad de obrar (MacIver, 1947:422).

El punto de partida de la teoría de la acción social de MacIver es el individuo, o mejor, la persona en cuanto síntesis de individualidad y sociabilidad. En esta visión la conciliación de los intereses del individuo y de la comunidad representa la condición primera de la estructura compleja de la sociedad civil; y es también la primera condición de la personalidad humana. Los valores que se persiguen en común encuentran siempre su realización en los particulares individuos (MacIver, 1947:429). De esta forma, el análisis de MacIver encuentra una concreta referencia en aquel ámbito particular que la acción social descubre cuando opera activamente en la esfera de la cultura, un ámbito en el que el Estado no puede intervenir completamente (MacIver, 1947:433).

El "liberalismo comunitario" de MacIver quiere evitar el doble error de las teorías individualistas y de las teorías organicistas. Gran estudioso de la comunidad, es consciente sin embargo de que los individuos no están integralmente ligados a un grupo; tienen necesidad de un grupo pero no necesariamente del grupo en que han nacido o de otro grupo particular. Para MacIver, "los hombres no pertenecen a un solo grupo sino a muchos grupos al mismo tiempo" (MacIver, 1933:25). Esto implica, especialmente en una sociedad compleja como la nuestra, definida por MacIver como multigroup-society, que no existe un grupo o una organización "que comprenda y circunscriba enteramente la vida social del hombre" (MacIver, 1933:284). Las teorías organicistas, que han rechạzado el "vacío individualista" por no mirar más allá del aspeẹto competitivo de la vida económica del hombre moderno y por ignorar "las profundas necesidades de las que se nutre el sustrato social", cometen el error de identificar la sociedad con una concreta forma asociativa, el Estado. Se trata de una identificación radicada en las teorías sociales de impronta organicista y funcionalista: una identificación que minusvalora que el agente social "sea una unidad auto-dirigida con un cierto grado o sentido de autonomía" y que la sociedad a la que pertenece "no le prescribe toda acción singular y, especialmente, no le prescribe todo su pensamiento" (MacIver, 1947:422). 
La espontaneidad y la creatividad del actor social y del grupo se deben confrontar con otros dos problemas conceptualizados por MacIver: por una parte, con la "necesidad de comunidad", es decir, de una unión satisfactoria con un único grupo y de un espíritu de colaboración que antaño animaba la vida de las sociedades grupo; y, por otra, sin embargo, con la creciente complejidad de la organización social, lo que equivale a la necesidad, hoy impuesta, de "pertenencias plurales" a diversos grupos al mismo tiempo. La necesidad de comunidad no puede ser hoy satisfecha persiguiendo el mito de la restauración de la sociedad con un único grupo, haciendo del Estado el centro, suficiente y necesario, de nuestra existencia moral y espiritual. Al mismo tiempo la multigroup society en la que piensa MacIver no puede ser una sociedad atomizada, individualista y tendencialmente anómica, y éticamente neutra, sino una sociedad plural de grupos, de comunidades, de asociaciones, de pertenencias (MacIver, 1947:439-440). Escondida tras esta tesis nos encontramos con otra no menos relevante que insiste en la necêsidad de salvaguardar la comunidad como lugar más apropiado para el crecimiento y la realización del actor social y de la espontaneidad de su acción (MacIver, 1947:198).

No es difícil comprender el motivo por el que el mismo MacIver había afirmado querer ser recordado por su compromiso en investigar sistemáticamente "los principios morales, sociológicos y filosóficos fundamentales de las instituciones y de los procesos democráticos" (Alpert, 1953:513). Consciente de que un "poder político incontrolado" es una auténtica maldición para toda sociedad, MacIver veía en la democracia la posibilidad de realizar una virtud: la de poner límites a toda forma de "absolutismo" del poder. En tal sentido, su sociología está al servicio de una concepción sustantiva de la democracia.

\section{EL CONTEXTO EPISTEMOLÓGICO}

El contexto epistemológico más próximo a la teoría de la acción social desarrollada por Robert M. MacIver es el llamado individualismo metodológico. En él, por una parte, descubre la posibilidad de evitar todo peligro conectado a cualquier forma de razón planificadora y, por otra, encuentra la posible solución a las relaciones entre sociedad civil, comunidad y Estado, que es uno de los motivos que inspiran su sociología cultural y política.

La crítica a toda forma de planificación social y a la atribución al Estado de un primado intelectual y moral sobre la vida comunitaria, se salda en Maclver con el reconocimiento de la "complejidad de los fenómenos sociales". De esta forma también en MacIver, como posteriormente hizo Boudon, el punto de partida es una versión individualista y neoweberiana del saber sociológico y de la autocomprensión con que se capta el sentido de la sociedad, por lo que el mismo saber sociológico se convierte "en un instrumento esencial mediante el que promover la creciente conciencia de esta complejidad" (Boudon, 1997:174). 
Para MacIver, como para Simmel, Alfred y Max Weber, el objetivo de la sociología no es primariamente descubrir las "regularidades", sino comprender (verstehen) la "motivaciones" del actor social. Sólo de un modo secundario MacIver se interesa por el estudio de las estructuras sociales, entendidas solamente como los ámbitos "reguladores" (tecnología base, esfera económica, esfera política) que en realidad dependen de la esfera de la "cultura" y a los que define como "ámbitos constructivos" del mismo orden social. Para MacIver, estos ámbitos siempre tienen como punto de partida las acciones de los individuos particulares (Boudon y Bourricaud, 1982:37).

La esfera de la acción social y la red de acciones e interacciones que se desarrollan en el interior de la vida social y comunitaria es para MacIver una esfera originaria y "constructiva" de la dinámica cultural de la sociedad. Se le reconoce una espontaneidad y una capacidad creadora que la intervención del Estado y de las instituciones deben simplemente reconocer y favorecer. Sin embargo, Maclver admite la existencia de una social causation: la explicación de la acción social y su comprensión puede tener lugar mediante un esquema causal. Por tanto, para MacIver, igual que como afirma Boudon de Weber, Simmel, Sombart y otros sociólogos ligados al paradigma del individualismo metodológico, la sociología, cuando utiliza métodos individualistas, puede desarrollar teorías cuya naturaleza lógica no es sustancialmente diferente de las teorías construidas por las ciencias exactas. Del hecho de que sólo las acciones elementales de los individuos capaces de dar cuenta de los fenómenos macrosociológicos, no se deriva que éstos "sean el producto del libre albedrío o de una libertad concebida como absoluta" (Boudon y Bourricaud, 1982:38).

De una acción social "nosotros medimos sus manifestaciones, pero continuamente revela nuevos aspectos, asume nuevas direcciones, entra en nuevas conjeturas que no preveíamos y que quizás no podríamos haber previsto" (MacIver, 1964:376). La propia dinámica intrínseca que caracteriza a todo proceso social y a toda acción que se desarrolla en su interior representa su elemento distintivo; pero también lo es aquella dimensión que revela la permanencia de un elemento de indeterminación intrínseco al que es necesario referir la idea misma de naturaleza humana. Y de esta última MacIver dice que nos encontramos ante el deber de "perseguir respuestas infinitamente cambiantes ante situaciones infinitamente cambiantes" (MacIver, 1964:376). Ello implica que si, por una parte, nos podemos aproximar al problema de la comprensión de la naturaleza humana "desde muchas perspectivas", por otra, debemos reconocer que la naturaleza humana posee una base de identidad en todas sus manifestaciones cambiantes y que estas perspectivas deben converger en un punto que jamás puede alcanzarse de forma definitiva (Boudon y Bourricaud, 1982:40).

Se trata de un contexto epistemológico de derivación weberiana, aunque con muchos aspectos de discontinuidad y originalidad. Para MacIver la pura medición no es, ni puede ser, el fin y el objetivo último de la ciencia, sino tan sólo un tipo de 
RIS

BEVISTA INTERNaCIONAL DE SOCIOLOGIA

№ 39, SEPTIEMBRE-DICIEMBRE, 2004

MANUEL HERRERA y PEDRO CASTÓN

acercamiento a ella. La crítica a la reducción de la sociología a sociografía y estadística no impide a MacIver reconocer cómo los procedimientos estadísticos son esenciales para el acercamiento a algunas formas de cambio social. Simplemente que el planteamiento cuantitativo debe integrarse con otros planteamientos.

La sociología de Maciver es comprensiva en cuanto que reconoce cómo la misma sociedad y su noción son "subjetivas" y no mensurables según un criterio objetivo único y absoluto. La sociedad, para MacIver, es pertenencia, comunidad, interdependencia, redes de relaciones entre grupos e internas al propio grupo. El elemento intrínsecamente individual y subjetivo de la realidad social, y por tanto de la ciencia social, que es comprensión de una comprensión, según aquel doble nivel hermenéutico intuido por las sociologías fenomenológicas, está sin embargo ligado al hecho de que allí donde existe un grupo siempre existe un objetivo, y donde existe una sociedad siempre existe una especie de unidad indivisible.

Como en Weber, en MacIver es evidente una reacción al modelo positivista de explicación causal unido a la idea de una matriz neokantiana según la cual la presuposición del conocimiento es la inagotable multiplicidad del dato empírico al que corresponde la labor ordenadora de un intelecto limitado. El conocimiento pleno de la realidad solamente es un fin jamás alcanzado. El conocimiento tan sólo refleja una parte de la realidad.

El científico social está encaminado a comprender el significado que el actor atribuye a la propia acción, por lo que para MacIver - siguiendo a Max Weber-el problema de la explicación de la acción social es, en primer lugar, el problema de su comprensión y comprensión de acontecimientos fuertemente condicionados por la individualidad, la libertad y la creatividad del sujeto individual o de grupos y comunidades dotadas de identidades y singulares peculiaridades. Por tanto, MacIver está interesado en captar la estructura intencional y teleológica de la acción social para posteriormente referirla a aquel sistema de constricciones y condiciones, de medios, de alternativas, que derivan de una situación social objetiva. En este sentido, su posición epistemológica no es subjetivista, como ha creído Merton. MacIver está convencido, como Max Weber, de que la estructura lógica de las ciencias sociales no puede reconducir los fenómenos a un sistema de leyes generales. Sin embargo, en sintonía con la Kultursoziologie de Alfred Weber, no limita su análisis a la estructura individual de la acción social, sino que apoyándose en los conceptos de "comunidad" y "asociación" y en la tripartición entre orden cultural, orden tecnológico y orden social, lo abre a la perspectiva típica de la sociología de la cultura y de la sociología de la historia.

El procedimiento que MacIver ubica en la base de su Social Causation (1964) es fundamentalmente de tipo comparativo e histórico-interpretativo. Ello no implica la exclusión de la explicación causal, sino la idea de que explicar causalmente es la investigación de "factores diferenciales" a partir de la confrontación entre situaciones similares, y no la reducción a leyes generales. Esta es una elección 
epistemológica que lleva a MacIver a "ubicarse fuera tanto de las perspectivas metodológicas pragmatistas y conductuales, como de la concepción neopositivista de la ciencia" (Rossi, 1975b:55). He aquí el porqué de una teoría sociológica contracorriente y no siempre comprendida en su "singularidad" (Rossi, 1975b:55).

La perspectiva epistemológica adoptada por MacIver es anti-mecanicista: en más de una ocasión subraya el error de cuantos interpretan el organicismo, la evolución orgánica, el obrar mismo de la mente, en términos de un "mecanicismo". Un organismo, a diferencia de una máquina, no está construido, sino que se desarrolla como una unidad: el conocimiento, la mente, la vida, tienen un rol dinámico que no puede ser minusvalorado por ninguna teoría de la acción. Es necesario subrayar cómo en la base de su posición fuertemente crítica con el neopositivismo, el pragmatismo y el mecanicismo biológico, está "la variedad y solidez de la cultura histórica" mitigada por la Scottish common sense philosophy (Poggi, 1962:10). Y también esto hace de la sociología de MacIver una disciplina que no radicaliza los límites disciplinares con la historia y la filosofía. En MacIver la sociología no es una disciplina autárquica, criticando por ello, el proceso de "profesionalización" que con frecuencia ha empobrecido su objeto de estudio, identificando de forma reductiva la sociedad con sus estructuras y liquidando el tradicional concepto de cultura con la complejidad de sus referencias a contenidos simbólicos y significados sensatos del obrar humano (Tenbruck, 1985:72).

De la sociología alemana clásica, MacIver comparte su interés por rechazar la tendencia a la "autarquía" de la sociología y, su vocación por utilizar el conocimiento de otras disciplinas. Por ello la sociología "comprensiva" de MacIver es también al mismo tiempo una sociología concreta y realista, en cuanto que es alimentada por un interés constante por la esfera política. El realismo que le guía está encaminado a evitar la construcción de una ciencia "que no sirve para nada". De ahí también su rechazo a dejarse condicionar por cualquier tipo de conformismo, rechazo que le ha llevado a "resistirse tanto a la sirena de la investigación de campo como al reclamo de la grand theory" (Poggi, 1962:9).

La posición epistemológica de MacIver acoge y, al mismo tiempo, supera la tradición weberiana en otro sentido Brecht ha hablado de un "relativismo científico", fundado en una rigurosa distinción entre juicios de hecho y de valor, sin que esto le impida tomar posición y "refugiarse en los errores de una Wertfreiheit para la que aquello que no puede probarse en cuanto perteneciente a la categoría de los valores es no existente o irrelevante" (Brecht, 1959:249).

La actualidad de la obra de MacIver también está conectada con ese intento de resolver el típico dilema del científico social, conjugando "honestidad científica" $\mathrm{y}$ "capacidad de resolver entre alternativas morales y políticas de nuestro tiempo" (Poggi, 1962:11). Un ejemplo de esta capacidad es su sociología política, de la que deriva una contribución relevante a su teoría de la acción social: ésta nace de la intuición del necesario primado de la acción social y de la red de acciones que constituyen las comunidades vivientes, incluso respecto al Estado, una asociación 
RIS

REVISTA INTERNACIONAL DE SOCIOLOGLA

NN 39, SEPTIEMBRE-DICIEMBRE, 2004

MANUEL HERRERA y PEDRO CASTÓN

que debe favorecer esta red, garantizando los derechos de todos. De esta forma el liberalismo de MacIver es, por una parte, la misma sustancia de su sociología política y cultural, y por otra, imprime una connotación precisa a su epistemología (Alpert, 1972:514).

\section{CONCEPTOS SOCIOLÓGICOS FUNDAMENTALES}

\section{Relación social y sociología}

La concepción de la sociología propuesta por MacIver está fuertemente conectada a la definición de "relación social". Tras una fase en la que dominó el "paradigma organicista", con el que la sociología había sido reducida a un pálido reflejo de la biología, se entró en una fase en la que fue entendida como "ciencia natural". El paradigma "naturalista", en concreto, presuponiendo que "la sociología como las ciencias físicas se interese por los objetos de la percepción, objetos susceptibles de ser registrados por medio de instrumentos, divisibles en unidad, susceptibles de ser sumados y sometidos a procedimientos de tipo cuantitativo" (MacIver, 1926:26), ignoraba la verdadera diferencia entre una relación física y una relación social. A partir de este desconocimiento fue posible, en el ámbito de la teoría sociológica de la acción, pasar de un extremo a otro: de las abstractas generalizaciones al rechazo de las teorías en nombre de la "sacralización del hecho"; de una ciencia social saturada de prejuicios teológicos y morales a una sociología "animada por el dogma revolucionario del behaviorismo"; de una ciencia que ignora la naturaleza del conocimiento a una que se funda "en una base de valores ya desgastados", hasta llegar a una ciencia "que trata las relaciones sociales como si existiesen objetivamente prescindiendo de los valores que las han creado" (MacIver, 1926: 26). Y esto por el hecho de que las ciencias sociales se enfrentan a dificultades que, sin embargo, están menos presentes en las ciencias de la naturaleza.

La sociología, en concreto, se encuentra ante fenómenos que implican una causalidad extrañay desconocida al mundo puramente físico; fenómenos que "en cuanto motivados soncreadosporaquellarealidad evasivay compleja, peroinnegable, queesla mentalidaddelhombre"(MacIver,1926:37).Conceptoscomo,porejemplo,eltrabajo,el Estado, la soberanía, el crimen, la desocupación, el folklore, la institución y las actitudes sociales, son siempre variables e indeterminados: todos, sostiene MacIver, presentan una "intangibilidad" que causa problemas al científico social. Es esta dificultad, esta intangibilidad (relativa) de los propios objetos de estudio, siempre ligados a una particular cultura, mentalidad y sensibilidad, lo que determina la constante tendencia a dirigirse hacia los paradigmas más exactos de las ciencias no sociales (MacIver, 1926:27).

Sin embargo, para McIver, es necesario proceder de forma totalmente opuesta: los fenómenos y los objetos de las ciencias sociales no deben adaptarse a nuestros 
métodos, sino que estos últimos deben adaptarse al material y al objeto de nuestro conocer. El behaviorismo radical es un típico ejemplo de una forma de observación que aplica los mismos instrumentos a materias diferentes. Al contrario, en el estudio de las acciones y de las relaciones sociales está aquello que estamos buscando y que debe determinar la forma en que lo buscamos. En este sentido toda ciencia tiene su propio camino, una propia senda hacia la verdad (MacIver, 1926:28).

En vez de refutar la importancia de una reflexión de carácter metodológico (véase la obra Social Causation), es consciente de que "una total inmersión en el método conlleva el riesgo de paralizar la capacidad de hacer preguntas" (MacIver, 1926:29). Una confianza ciega en el método tiene como corolario no sólo la creencia en los "hechos", sino la necesidad de descubrir nuevos. Por tanto, la sociología no esquiva la necesidad de asumir el arduo objetivo de la interpretación: la especulación teórica no debe ser eliminada ni reducida a un apéndice de la investigación empírica, más bien debe ser "controlada". El mismo ejercició del conocer sociológico es una acción social a la que es propia la dimensión esencial de la interpretación y la imaginación (Maciver, 1926:33).

Sin embargo, el estudioso de la acción social no puede tener en cuenta solamente aquel inner system que constituye el agente social. Esto también traslada a una dimensión externa que entiende como "situación social". Para MacIver toda situación social consiste en la adaptación de un sistema interno a un sistema externo de realidad. Existe un inner system, un complejo de factores ambientales que ofrece los medios, las oportunidades, los obstáculos, las condiciones respecto a las que se adapta el sistema interno. La relación entre un sistema interno y otro externo determina la diferencia esencial entre una relación social y una relación física, entre ciencias sociales y ciencias naturales, desde el momento en que estas últimas solamente contemplan un orden exterior.

La interpretación de la acción social necesariamente conlleva el riesgo de incurrir en una forma de "intuición": los métodos y los modelos siempre son útiles en sociología, pero no pueden sustituir a una forma disciplinada de imaginación. De este conocimiento deriva para la sociología un inevitable estatuto de incertidumbre, de provisionalidad, de cautela, de humildad, un estatuto contrapuesto a toda concepción constructivista. MacIver expresa esta convicción recordando cómo el mismo lenguaje sociológico es, por su naturaleza, abstracto en función de la relativa "intangibilidad" de los fenómenos sociales.

\section{Sociedad, comunidad, intereses}

Los hombres expresan y realizan su "sociabilidad" mediante la creación y recreación de una organización con la que guiar y controlar su conducta en una amplia variedad de formas. Para Maclver esta organización es la "sociedad", que al mismo tiempo "libera" y "limita" las actividades del hombre, fijando los estándares a seguir y a los que adaptarse. A pesar de las imperfecciones y las tiranías 
RIS

REVISTA INTERNACIONAL DE SOCIOLgGta

$\mathrm{N}^{\circ} 39$, SEPTIEMBRE-DICIEMBRE, 2004

MANUEL HERRERA y PEDRO CASTÓN

que tal organización ha manifestado en el curso de la historia, siempre permanece como "una condición necesaria" para la misma realización de la vida del hombre. Para MacIver la sociedad es un sistema de usos y procedimientos, de autoridad y ayuda recíproca, de reagrupamientos y divisiones, de control de la conducta humana y de libertad, pero también es la red de las relaciones sociales, una red que cambia continuamente construyendo el changing pattern, el modelo cambiante de tales relaciones sociales. Sin embargo, a este aspecto normativo y constrictivo corresponde una segunda característica. Para MacIver, y en esto insiste bastante, solamente existe la sociedad allí donde un ser social actúa en las confrontaciones con el otro de forma que está en primer lugar determinado por "el reconocimiento del otro". El reconocimiento del otro y el reconocimiento recíproco constituyen para MacIver la raíz de la sociabilidad y una especie de "condición psíquica y cultural" sin la que no puede nacer y mantenerse una relación social.

La sociabilidad es un atributo fundamental de la naturaleza humana. Pero, se pregunta MacIver, ¿en qué sentido pertenecemos a la sociedad?, ¿en qué sentido la sociedad nos pertenece?, y, sobre todo, ¿cuál es la naturaleza de nuestra dependencia hacia ella? El problema de la relación entre individuo, grupo y sistema social constituye el "foco" de la investigación sociológica, cuya fecundidad es medida por su contribución al problema de la relación entre individuo y sociedad. En este sentido, existen dos planteamientos unilaterales que MacIver rechaza. Por una parte, aquel que emerge de la línea de pensamiento que va desde Hobbes a Smith y que entiende la sociedad como medio para la protección del hombre contra las consecuencias de su especie. Por otra, la teoría organicista que se explicita concretamente en el positivismo comtiano y durkheimiano, y que MacIver conecta con una línea de pensamiento que va desde Platón a Hegel para terminar en Spengler, línea que considera a la sociedad como un organismo a partir de un proceso de identificación con una idea común. A la inadecuación de estas dos concepciones, MacIver contrapone el principio de una relativa armonía entre individuo y sociedad, principio que no puede implicar la reducción de un término al otro.

La sociedad es para MacIver sinónimo de una realidad originaria que no puede ser anulada ni confundida con el Estado. El elemento social no puede equipararse ni puede coincidir con el político: tal confusión, dice MacIver, altera toda posibilidad de comprensión tanto de la sociedad como de las diferentes instituciones socio-políticas. Para comprender la sociedad en lo concreto de su existencia es necesario prestar atención a uno de sus componentes, la comunidad.

Para MacIver el sujeto de estudio de la sociología es la comunidad; aún más, la sociología puede definirse como "la ciencia de la comunidad" (MacIver, 1928: 48). Mientras que las otras ciencias sociales estudian las particulares actividades asociativas que se desarrollan en el interior de la sociedad (por ejemplo, la economía), la sociología estudia estas actividades como aspectos de la vida común (MacIver, 1928:53). Para la necesidad de este estudio sintético de la comunidad está la sociología. 
La comunidad es una unidad espiritual, pero no en el sentido de la integridad indisoluble de un espíritu único: más bien en el sentido de una comunidad de espíritus ligados por relaciones sociales. Estas dos formas de unidad son totalmente diferentes: una comunidad es la unión de espíritus, pero ella misma no es un espíritu. Para MacIver, la hipótesis durkheimiana de una mentalidad colectiva supra-individual debe ser rechazada: una "mente colectiva" sólo es el fruto de un "contagio psíquico" que destruye, no crea. La comunidad es, sin embargo, una red de semejanzas y de diferencias, de aquello que es común y de lo que es diferente en sus miembros. MacIver la define como un sistema "complejo y maravilloso", para no recurrir a la hipótesis de una "mente común". Los ligámenes de la sociedad están en los miembros de la misma sociedad; en la comunidad no encontramos ni una suma de individuos, ni una suma de partes. Al contrario, las propias relaciones sociales de cada individuo no están fuera de él, sino que son "revelaciones" de su personalidad. "La unidad en la que estamos pensando no es de tipo mecánico, ni orgánico, ni psíquico: solamente puede llamarse con el término de comunal" (MacIver, 1928:92).

El término comunal define aquella particular cualidad del ligamen social desconocida por la línea de pensamiento que va desde Fichte a Comte y en la que la sociabilidad coincide con una sola virtud, a saber: la autonegación, la obediencia y la observancia ciega de la tradición (el servicio a un país, a una raza, dice MacIver, no puede constituir el fin último de nuestra vida). Por el contrario, MacIver habla de "persona social" como de la única verdadera de la auténtica "unidad" de la que se puede hablar, mientras el resto son relativas y secundarias (MacIver, 1928:94). Igualmente es desconocida esta cualidad por la línea de pensamiento que va desde Trasimaco a Nietzsche: una forma de "amoralismo" que en Nietzsche, observa MacIver, está ligada a una auténtica y noble idea del valor de la individualidad que se autodetermina, aunque concluye en una posición extrema y contradictoria. En cuanto que considera la relación social por aquello que no es, la posición de Nietzsche es, en este sentido, una reacción al sociologismo positivista; no comprende que las relaciones sociales no son vínculos externos, ni necesidades en las que la personalidad se desarrolla, sino, por el contrario, "funciones de la personalidad de cada uno, cuya realización coincide con su propia realización personal" (MacIver, 1928:95). Sólo en este sentido el interés de la sociedad coincide con el interés de cada uno de sus miembros. Sólo en la sociedad la personalidad puede encontrar una morada en la que desarrollarse, y sólo en el servicio a la sociedad el individuo puede atender a la realización de la propia vida. En este sentido el interés de la sociedad y el interés del individuo coinciden. El mismo concepto de interés en MacIver no aparece tan en contraste con el de comunidad como la distinción realizada por Tönnies en tal sentido.

Dice Giner que lo importante en la distinción tönniesiana entre Wenssenwille (voluntad natural) y Kürwille (voluntad racional-instrumental) es la manifestación de una polaridad y tensión permanentes. "Su esfuerzo va dirigido a mostrar cómo 
R IS

REVISTA INTERNACIONAL DE SOCIOLgGía

ambas formas de expresión surgen una de la otra en el proceso del desarrollo cultural e histórico, cómo se implican dialecticamente y cómo no son siempre separables del todo" (1979: 12). Maclver distingue en el ámbito de la acción humana dos factores polares (polar factors), factores que él considera distinguibles analíticamente, pero indivisibles en la concreción del obrar: en sentido objetivo la acción está conectada al "interés", en sentido subjetivo a la "voluntad". Dos factores siempre ligados por una precisa correlación: toda realización de interés del hombre siempre es una relación activada por la voluntad. El término "interés", entendido como motivo-fuerza, es preferido por Maclver al de "fuerzas sociales" (demasiado indistinto) o al término "deseo", (demasiado subjetivo) o a las expresiones "intención" y fin, en cuanto que muy ligadas "a los objetos racionales de la voluntad" (MacIver, 1928:29). El término "interés" viene de esta forma a constituir uno de los referentes centrales de la teoría de la acción social de MacIver (MacIver, 1928:102). Siempre el interés está en el trasfondo de la actividad humana de los cambios sociales. Los intereses aumentan y se diferencian: algunos son eternos y otros cambian y pasan, y de este modo transforman las asociaciones que ellos mismos han creado.

Entre "interés" y "comunidad" existe una concreta correspondencia: para MacIver una comunidad alcanza su existencia por el hecho de que los intereses sólo son realizables en la vida en común. Por tanto, representan una "emergencia" de la comunidad; y de ahí que, una clasificación de los intereses constituya una necesaria introducción al estudio de la propia comunidad.

El concepto de interés es fundamental para comprender la formación de las instituciones democráticas modernas, un proceso en el que se han confrontado dos concepciones de "interés" sustancialmente diferentes (aunque ambas nacidas en oposición al sistema feudal). El sistema feudal de linajes era un sistema de intereses regulado por el dominio de la propiedad sobre la tierra. El crecimiento de otras formas de propiedad significó, en la época moderna, una modificación del sistema, pero no necesariamente del principio. La Revolución Inglesa de 1688, por ejemplo, tan sólo supuso que el antiguo principio de que la propiedad gobierna el país se ampliará a la clase que se estaba afirmando y en una situación diferente. Locke y Harrington no fueron, en ese sentido, profetas revolucionarios, sino nuevos intérpretes de la antigua doctrina del rol político de los intereses de la propiedad. Sin embargo, existe una segunda línea de interpretación de la idea democrática que, sin embargo, se ha afirmado negando el principio del interés: "ésta — dice MacIver- ha traducido la doctrina de los derechos naturales en la máxima autoevidente de que todos los hombres son iguales y que de ella se deriva una conclusión que Locke y otros habían rechazado" (MacIver, 1932:144). Se trata del principio enunciado "con una lógica intransigente" por Rousseau. El principio que inspira la Declaración de los Derechos del Hombre y que en la Francia revolucionaria deroga todo interés de linaje (todo corporate interest). 
El conflicto entre estas dos doctrinas se abrió en los siglos sucesivos y es resultado de los acontecimientos derivados de la Revolución Americana. Se refleja, dice MacIver, en el tránsito del lenguaje de la Declaración al de la Constitución. Se reconoce y sanciona que el conflicto de intereses crea el problema del gobierno: es el origen de las facciones y de los partidos, y, por tanto, de la misma política. Sin embargo, los continuadores de Rousseau, que MacIver identifica con la "escuela de Fichte" y con Hegel, desarrollan la tesis opuesta e insisten en la subordinación de los grupos de interés a los fines universales del Estado. Es decir, éstos desconocen aquello que para MacIver es un dato sociológico incontrovertible: las cuestiones políticas inevitablemente están determinadas por la lucha entre los grupos de interés. En este sentido, el Estado debe ser entendido como una agencia, una organización que determina el ajuste de los intereses; su poder es el de regularlos en relación con un continuo ajuste para preservar una "armonía práctica". De esta forma, en el ámbito del análisis sociológico, el concepto de interés puede explicar muchos fenómenos sociales. En concreto, a MacIver se le debe la propuesta de distinguir entre actitudes y estados de conocimiento, por una parte, e intereses, 0 bien los objetos hacia los que estos estados están dirigidos, por otra. Si el término "interés" es utilizado con este significado objetivo, el concepto es particularmente útil para explicar el nacimiento y el cambio de la organización social. Al avanzar el proceso de diferenciación y aquel que MacIver llama proceso de civilización, los intereses siempre se organizan progresivamente en "asociaciones" y de esta forma se hacen "más específicos, definidos y limitados".

La referencia al concepto de "interés", sin embargo, no agota la definición que MacIver ofrece del concepto de "comunidad". "Existe comunidad allí donde los miembros de un grupo, grande o pequeño, viven juntos de tal forma que comparten no este o aquel interés, sino las mismas condiciones-base de la vida en común" (MacIver, 1932:144). El aspecto característico de la comunidad es, por tanto, el hecho de presentarse como un terreno de vida común con un cierto grado de coherencia social. En concreto, para MacIver dos son las bases de la comunidad: la locality, es decir, el ligamen con un territorio, y el community sentiment, un sentir común.

Una comunidad siempre ocupa un área territorial. Por tanto, la comunidad es un lugar, una configuración física: sólo desde la ocupación de un territorio se desarrolla un área de vida común y un sentimiento común. Sin embargo, a la configuración física también corresponde una psicológica que coincide con lo que MacIver define como "sentimiento comunitario", en cuanto que implica la idea del other-regarding, del altruismo, de la atención al otro contrapuesto y a un self-regarding, al sentir egoísta. Este es un aspecto que aproxima decisivamente la posición de MacIver a la de Tönnies. En concreto, el sentimiento comunitario combina en sí algunas actitudes y elementos interconectados (MacIver, 1933:62):

1. El primer elemento es aquél que viene definido por el we-feeling, por el sentimiento del nosotros. 


\section{RIS}

2. Posteriormente está el role-feeling, el sentido de un rol específico y función que cada uno desarrolla en el interior de una comunidad.

3. Estrechamente conectado con el precedente, está la dependency-feeling, un sentimiento de dependencia a la comunidad como condición necesaria de la vida del individuo.

El sentimiento de identificación, el de rol y el de dependencia se manifiestan en grados diferentes y en combinaciones diversas en las distintas comunidades. Particulares modos de obrar, costumbres, tradiciones, formas de hablar, siempre son las señales de una vida efectiva en común en la que, sin embargo, siempre desde la concepción de MacIver, cada actor social participa activamente elaborando una aportación creativa propia.

Posteriormente, para comprender plenamente el concepto de "comunidad" es necesario confrontarlo con el de "asociación". Las asociaciones son los órganos de la comunidad, es decir, los medios para conseguir determinados y específicos fines (MacIver, 1933:13). Por tanto, la asociación es una organización en el interior de la comunidad, que surge libremente, mediante un pacto. La pertenencia a la asociación tiene un significado social limitado al fin específico por el que ha sido creada. Frecuentemente confuso con la idea de comunidad, el Estado es para MacIver un tipo de asociación. El Estado es una "agencia", aunque de rango más elevado. En algunos casos, el Estado intenta llevar a cabo un pleno control de las comunidades que viven en su interior y de todo aspecto de la vida humana. Ahora bien, incluso cuando realiza plenamente este control, no puede convertirse en una comunidad, sino sólo "en una asociación que controla la comunidad" (MacIver, 1933:134). De igual forma, el ser social y humano no agota su significado en el "ser ciudadano" que, según MacIver, sólo es un rol entre los muchos que se ejercen en cuanto seres humanos. En este sentido, las instituciones sociales y políticas tan sólo son una de las formas establecidas de procedimientos características de la actividad del grupo.

\section{Evolución y progreso social}

Para comprender a fondo la teoría de la acción social de MacIver, no menos importantes, son las reflexiones que dedica a los conceptos de "progreso" y "evolución". El error de confundir estos dos aspectos de la dinámica social, típico del positivismo de Comte y de Spencer, tiende a comprometer el carácter científico, dice MacIver, de muchos análisis sociológicos. En concreto, el concepto de progreso aparece con un significado variable y no conclusivo, indeterminado, diferente para los diversos individuos, épocas y grupos sociales (MacIver, 1933: 411). Cuando el sociólogo lo identifica con la idea de un completo funcionamiento de una personalidad integrada, no consigue evitar la ambigüedad de la idea de funcionamiento: en el sentido de que todo funcionar-observa MacIver-siempre 
es selectivo y cada uno define aquello que funciona no sólo en términos extensivos, sino también en términos cualitativos. Por tanto, el concepto de progreso no tiene, ni puede tener, una aceptación universal. Preguntarse: iprogreso en qué términos?, siempre implica inevitablemente la referencia a un reino de los valores últimos.

Aquí MacIver alude a la conciencia crítica que siempre debe acompañar al sociólogo. Por una parte, la sociología y el análisis de la acción social son ubicadas ante los valores; por otra, no les corresponde el objetivo de establecer los fines y los valores últimos. Si bien la Wertfreiheit es para MacIver un criterio metodológico irrenunciable, en el sentido de que la ciencia social debe abstenerse de confundir el plano de los hechos con el de los ideales, considera necesario que el análisis sociológico sea guiado por la conciencia de que la acción social y la vida de las comunidades está orientada por un significado y, en algunos casos, por la orientación a un valor (MacIver, 1933:417). Esta conciencia y, al mismo tiempo, un adecuado distanciamiento crítico permiten comprender al sociólogo que el concepto de progreso no es una invención moderna (MacIver, 1933:418).

\section{EL PROBLEMA DE LA ACCIÓN SOCIAL}

Por tanto, la categoría que guía el análisis sociológico de Maciver no es la de progreso, sino la más amplia de "evolución", que coincide con la de cambio social. MacIver es un neoevolucionista crítico de los esquemas de Spencer, pero neoevolucionista.

En su interior puede haber progreso, pero también regreso y decaimiento. La sociología de MacIver es "comprensiva" en la medida en que deja a un lado el problema de una interpretación total de la evolución general de la sociedad humana (como en el positivismo) para concentrarse en un nuevo objeto de estudio que no es tanto el hecho social como el valor y los intereses que sustancian al hecho social y le atribuyen significado. La acción social se sustancia de imaginación, intencionalidad respecto a los intereses y valores culturales. La sociología es una ciencia comprensiva en cuanto que traslada, incluso para MacIver, a un doble círculo hermenéutico (MacIver, 1926:35).

En el centro de la teoría de la acción social de MacIver está el concepto de "valoración dinámica", un concepto que aplica no sólo al actor social, sino también a sujetos más amplios, y que traslada explícitamente a otras formulaciones similares como la de W. I. Thomas, que planteó la "definición de situación". Existen defining agencies, por ejemplo una familia, una comunidad y, en general, toda entidad social en situación de alcanzar una común "valoración de situación". El problema es comprender la forma en que los factores de los diversos órdenes estructurales están selectivamente unificados en los procesos de comportamiento individual y de grupo. La pura clasificación de estos factores (como veremos, por 
una parte, giran en torno a la distinción que realiza entre un cultural order, un technological order - también llamado civilización-y un social order, y, por otra, entre esfera física, orgánica y consciente-intencional) "revela un problema, pero no elabora una respuesta" (MacIver, 1936:35). Según MacIver, la clasificación realizada por Alfred Weber (1931:84), por ejemplo, no aborda suficientemente el problema de la social causation, de la relación que se construye entre estas esferas u órdenes y la acción social. Sin embargo, el problema es comprender cómo todos estos factores, en toda situación específica, entran en un acto consciente, en una conjugación dinámica (MacIver, 1964:295). Para tratar de explicar esta interacción entre diferentes órdenes estructurales y acción consciente, MacIver define el concepto de dinamic assessment (de valoración dinámica).

En toda acción social, individual y de grupo, está presente una "valoración dinámica", es decir, un principio organizativo de amplio radio, en el sentido de que "la coherencia, la consistencia o el grado de integración alcanzado por cada uno de los tres órdenes (cultural, de civilización, social) de la esfera consciente es obra de continuas y sucesivas valoraciones dinámicas individuales y de grupo" (MacIver, 1964:320). En este dinamic assessment se desarrolla "una ponderación de factores que no siempre utiliza escalas objetivas, sino que varía con todo estado de ánimo y con toda situación. Allí donde hay ponderación, ésta es relativa a un esquema de valores" (MacIver, 1964:327). Pero la acción social no puede comprenderse solamente a partir de una referencia a los valores, al ligamen con el cultural order. Ésta encuentra límites precisos y resistencias en el más amplio contexto social en que se desarrolla, aplicando a ellos la lógica de la relación medios-fines.

En la acción social puede ser individuada una específica "causa" (que jamás es la única, pero es la más evidente) que MacIver llama "incentivo", es decir, todo factor subjetivo considerado en cuanto inicia y dirige el curso de una acción significativamente asociada a un factor subjetivo. Allí donde explicamos el comportamiento humano en los términos de "fuerzas", "instintos", "emociones" o "motivos", según MacIver, apelamos a los incentivos. El concepto de "incentivo" traslada al de intencionalidad, en cuanto que el agente tiene conciencia de la dependencia de la acción de su propio impulso. Este aspecto subjetivo o psíquico es un dato primario del comportamiento de los seres sociales. Sin embargo, la sociología debe preguntarse: "¿hasta qué punto y en qué condiciones estamos justificados para escoger un particular atributo subjetivo como explicación de la actividad individual o de grupo?" (MacIver, 1964:220-221). Ahora bien, el concepto de "incentivo", y una explicación social que se límite a él, tiende a prescindir de la relación medios-fines, en cuanto que no se refiere a los medios, a un objetivo externo, a un elemento no-subjetivo. Por tal razón, MacIver introduce la distinción entre "objetivo" y "motivo subjetivo" (MacIver, 1964:221).

El "motivo subjetivo" está en el origen del significado de muchas cosas, que con frecuencia nos desvelan los pensamientos escondidos que las han inspirado 
y no el obrar o el hablar. Aquí el incentivo jamás está dado, siempre es inferido. Para MacIver "cualquiera que trate de explorar la naturaleza humana o de explicar las acciones sociales está inmerso en este problema -el novelista, el dramaturgo, el biógrafo, el historiador, el antropólogo, el psicólogo social, el sociólogo, el filósofo social" (MacIver, 1964:222). Son situaciones en las que la acción está completamente a merced de las fuerzas internas de este tipo. Por ejemplo, el llamado crimen pasional o el amplio campo de los sentimientos: la gratitud, la envidia, la confianza, los celos. Respecto a los llamados "objetivos" adoptados para la explicación de las acciones sociales, son ilimitados. Sin embargo, estas fuerzas internas, estos incentivos, son limitados y definidos.

Un incentivo no puede ser identificado con un "instinto". En general, el intento de hacer derivar las acciones sociales de un conjunto de instintos es posible para el mundo animal, pero no para el mundo de los humanos. Un instinto - ésta es la dificultad - solamente puede ser el nombre para las uniformidades, pero no una clave que explique las diferencias. De ahí el fracaso de muchos intentos de dar una definición completa: por ejemplo el de Jung, con sus tipos psicológicos; el de Freud, que ubica el instinto sexual como fuerza eje del comportamiento; el de Thomas y Znaniecki (con sus cuatro deseos: de correspondencia, reconocimiento, seguridad, nueva experiencia), y sobre todo el de Pareto (y sus seis clases de residuos), que MacIver considera los más interesantes y elaborados. Se trata de intentos de explicación de la acción que ofrecen esquemas de imputación causal útiles, pero que comparten una dificultad: la base de la clasificación siempre es oscura, el elenco de los instintos o de las tendencias siempre es diferente, y cada uno de ellos, si se ha captado a la luz del otro, resulta incierto y dudoso (MacIver, 1964:224).

La conclusión que MacIver extrae del análisis de las fuerzas subjetivas, de los "incentivos" de la acción, es útil para comprender su crítica a toda explicación monocausal. Un incentivo, un instinto, ofrece una indicación respecto de la dirección dinámica de la personalidad, pero en la personalidad adulta el incentivo a la acción es un impulso complejo y eternamente variable del yo dinámico (MacIver, 1964:226).

En el análisis de la acción social, los aspectos subjetivos e instintivos necesariamente deben estar conectados a un contexto objetivo, a una situación social objetiva, aquélla en la que el ser social se encuentra y actúa (MacIver, 1964:226). Incluso si el análisis de cualquier relación o acción social jamás puede confiarse a los simples datos perceptivos, siempre es fruto de una inferencia que implica una apelación a la razón. En la acción social, por ejemplo, la imputación de los motivos nunca puede llegar a una demostración completa (MacIver, 1964:227). Sin embargo, una dificultad no puede significar la renuncia a la imputación de los motivos registrada como cuestión no-científica. La sociología, dice MacIver, no puede renunciar al problema de la inteligibilidad de la realidad (MacIver, 1964: 228).

De esta forma, el científico social no puede detenerse en el estudio del "cómo" de la acción social, de las condiciones en que se verifica y de las probabilidades 
de su manifestarse en aquellas condiciones, ya que entre las condiciones del obrar humano existen "actitudes subjetivas e impulsos", en ausencia de las cuales tal obrar "no sería ya humano" (MacIver, 1964:228). Las fuerzas sujetivas son las condiciones para explicar la acción social: por ello, dice MacIver, la pregunta respecto al "porqué" es más importante que el interrogante respecto al "cómo". No existe área de investigación social y humana a la que la cuestión del "porqué" sea extraña. La sociología no puede renunciar a la pretensión de conocer sólo el "cómo", también el "porqué" cambian las costumbres, aumentan los divorcios, disminuye la tasa de natalidad, surgen conflictos, nacen o decaen movimientos sociales.

Incluso la "desconcertante incertidumbre", que con frecuencia caracteriza al conocimiento, oscuro y elusivo, que posee el agente social de los motivos del propio actuar, no puede detener el esfuerzo de llegar hasta el fondo en el análisis de la estructura de la acción social. Aun en una lógica de aproximación, de búsqueda de evidencia que jamás coincide con la certeza absoluta, el sociólogo puede estudiar la acción social a partir de una tipología de las acciones sociales que se presenta como una amplia área de la ciencia social no suficientemente desarrollada. En concreto, una teoría de la acción puede conseguir el objetivo de comprender "la motivación característica de ciertos tipos de situación social" (MacIver, 1964:233).

El único error en el que puede incurrir la sociología es el de reivindicar una "certeza injustificada" y viceversa, el pleno reconocimiento de las dificultades en que incurre cualquier teoría de la acción social debe convertirse, dice MacIver, en la base de una exploración más amplia. Desde el punto de vista sociológico "debemos indagar en los impulsos que nacen de los movimientos sociales, los sentimientos que caracterizan la diferentes formas de solidaridad de grupo, los fuegos de las afecciones emotivas en diferentes condiciones sociales" (MacIver, 1964:237). Por ejemplo, dice MacIver, existe una "sociología de la emoción" que aún es completamente ignorada y que podría dar luz a los problemas de la motivación de la acción social.

\section{NEXO TELEOLÓGICO Y SOCIAL CAUSATION}

En la acción social está presente un impulso teleológico que se refleja en la conciencia de la misma acción y en su dinamismo. Tal impulso teleológico, que acompaña a la dimensión intencional y consciente de la acción, constituye, dice MacIver, la cualidad distintiva de la causation en la esfera socio-psicológica. A la presencia de un "porqué", del que se es consciente en el momento de obrar, está conectado el mismo concepto de causation, al que MacIver dedica su obra más notable.

$\mathrm{Si}$ en la esfera física la investigación de un porqué es investigación de un "principio invariante", en la esfera orgánica y social la investigación de un porqué es investigación de un "motivo". Se trata de una relación que es definida 
por MacIver como "nexo teleológico" de la acción social. Ahora bien, es preciso realizar una pequeña matización. MacIver no esconde que la comprensión de este nexo no agota la complejidad de la acción social (MacIver, 1964:79). El "nexo teleológico" se puede articular entre tres aspectos a los que MacIver llama el "porqué del objetivo", el "porqué de la motivación" y el "porqué del modelo". El porqué del objetivo es típico de toda forma de "racionalidad instrumental": en él, "lo mejor" de la acción coincide con el mismo concepto de "utilidad". El objetivo es una especie de "resultado-fin" que puede ser atribuido a cualquiera, incluso a todo tipo de asociación, allí donde, sin embargo, el "porqué en cuanto motivación" siempre es un aspecto de la personalidad del agente y no algo que él "hace" o "permite realizar". Con frecuencia, observa MacIver, el porqué en cuanto motivación yace, oscuramente, tras el porqué del objetivo. A estos dos diferentes "porqué" corresponden aquellos que MacIver llama orden cultural y orden tecnológico (MacIver, 1964:81). El tercer aspecto de la estructura teleológica de la acción social es definido por MacIver como el "porqué del modelo". Existe una amplia variedad de actividades en las que los hombres siguen "modelos predefinidos", modelos socialmente impuestos o culturalmente aceptados. El nexo está aquí entre una copia y un modelo. El modelo se convierte de esta forma en una especie de esquema general ejemplificado en un producto cultural, en un instrumento útil, en una forma de comportamiento. Corresponde al concepto aristotélico de causa formal. Una pregunta fundamental es por qué un modelo es elegido, por qué está investido de un valor práctico, o bien estético, social, etc.

Ahora bien, junto al nexo puramente teleológico, es necesario distinguir un nexo que MacIver define como "social", cuya investigación corresponde al específico objetivo de las ciencias sociales. No es posible responder en términos de "objetivo", "motivación" o incluso de "modelo". Ninguno quiere, observa MacIver, que exista una legislación social en la que se prescriba que una tasa de mortalidad decreciente esté asociada a una tasa demográfica decreciente. Más bien, estos fenómenos son "resultados sociales" de numerosas acciones individuales o de grupo dirigidas a los objetivos más diversos, pero que conjuntamente concurren para que sucedan. Para MacIver, ésta es la forma en que se crea la estructura social. También para nuestro autor la acción social tiene "efectos no intencionales", resultados no controlables perfectamente. Toda concepción ingenieril y planificadora tiende a minusvalorar esta característica fundamental de la dinámica socio-cultural. Esto también vale para los modelos culturales, para los hábitos y costumbres consolidados (MacIver, 1964:83). La creación de un modelo de comportamiento nace de la combinación de actividades diferentes. Por tanto, el nexo de causalidad social posee una propia especificidad que lo distingue tanto del "nexo físico", como del estrictamente "teleológico" (MacIver, 1964:83).

El análisis de la acción social va en la dirección no sólo de una acentuación del momento individualista e intencional, sino al mismo tiempo se encamina a subrayar la complejidad de los procesos sociales, su imprevisión relativa, el peligro 
RIS

REVISTA INTERNACIONAL DE SOCLOLgGía

No 39, SEPTIEMBRE-DICIEMBRE, 2004

MANUEL HERRERA y PEDRO CASTÓN

y los límites de su "planificación". De esta forma, el subrayar la complejidad de los fenómenos sociales va acompañado de la convicción de que es posible individuar nexos de causalidad social sin con ello olvidar como momento crucial del análisis sociológico la comprensión de las motivaciones del actor social (Boudon, 1997:158).

El amplio análisis de los conceptos de "voluntad", "intención", "interpretación" realizado por MacIver camina en la dirección de completar al agente social como factor causal distintivo. El ser consciente tiene voluntad, ya que muestra la voluntad resoluta de actuar a lo largo de ciertas directivas, y refleja una intención en la medida en que su voluntad tiene una dirección. Por tanto, el nexo teleológico está en la base de la acción individual y social al expresar el carácter de la persona consciente en cuanto dinámica. De esta forma, observa MacIver, si una actividad es querida, intencional, conscientemente tomada, el agente tendrá una cierta pre-cognición de lo que está por hacer y una anticipación dẹl estado de las cosas que cambia como consecuencia de su obrar. Por otra parte, señala MacIver, "el agente consciente siempre entrevé al menos dos alternativas: una es aquella que existe, perdura o brota si no interviene; la otra es la situación en cuando modificada por su intervención (...). En este sentido se puede decir que el agente inicia el cambio, ya que su elección determina cuál de las dos o más potencialidades serán actualizadas" (MacIver, 1964:248).

Dentro de los límites fijados por la situación o por los medios a disposición, la forma de actuar del actor social es abierta y no pre-determinada por nada externo, en el sentido de que "escoge internamente a estos límites en los términos de sus intereses y de sus actitudes" (MacIver, 1964:248). Los intereses y las actitudes son valoraciones que MacIver liga a la esfera de los valores. A partir de ella realiza la distinción entre el concepto de responsabilidad individual y responsabilidad social (MacIver, 1964:249).

El problema de la relación entre determinismo-indeterminismo, libertad-causalidad, encuentra en MacIver una solución particular. La pregunta respecto a la libertad de la voluntad va formulada en los términos de la "libertad de la persona que ejerce la voluntad". Y en tal sentido toda acción social es tanto libre como obligada. Libertad de la voluntad y ley científica no están en contraposición: el principio de libertad no niega el de causalidad (MacIver, 1964:251). El obrar social no podría ser libre si escapase al nexo causal, mientras que fuera de él todo obrar resultaría "incomprensible, caótico, vacío". Actores sociales y grupos son "participantes dinámicos" en el interior de un orden causal. En la realidad concreta de la situación en que vive, el actor social actúa según la comprensión, siempre imperfecta, de la situación social, dice MacIver, y su control siempre es limitado. $\mathrm{Su}$ acción modifica la situación, y la naturaleza del cambio depende de lo que él y la situación son. El actor social de MacIver se encuentra entre el equilibrio existente entre responsabilidad personal y responsabilidad social, entre libertad y determinación (MacIver, 1964:252). Por tanto, en la acción está la libre elección entre alternativas, pero no es libre la elección de aquéllas que deberían serlo. 


\section{LA ACCIÓN SOCIAL Y EL ORDEN CULTURAL, TECNOLÓGICO Y SOCIAL}

La esfera física, la esfera del ser orgánico y la esfera del ser consciente constituyen, para MacIver, las tres grandes esferas dinámicas en que se articula el mundo que nos circunda. En el interior de la última esfera, la consciente, distingue tres órdenes: cultural, tecnológico y social.

El que se limita a estudiar la esfera física busca leyes invariables y excluye, por principio, la cultura humana y las relaciones sociales (MacIver, 1964:279). $\mathrm{Al}$ contrario el científico social: su perspectiva está alejada totalmente de la del físico, en cuanto que "sus fenómenos dependen de y participan de la naturaleza del resto de órdenes del ser (...). No existen independientemente de la esfera físico-química. Se encuentran en estrecha relación con las condiciones de la vida orgánica. Están íntimamente asociados a los modelos culturales, las disposiciones, los instrumentos y las técnicas puestas a disposición por todas las ciencias" (MacIver, 1964:280).

Al intentar explicar adecuadamente la relación de causalidad que liga acción y ambiente social, MacIver elabora aquella distinción que, según muchos críticos, constituye uno de los aspectos más originales de su sociología. Se trata de una distinción cuyo único precedente teórico está en la Kultursoziologie de Alfred Weber (1927 y 1951).

Según MacIver, el análisis de la acción social debe, en primer lugar, comprender el significado de las características y componentes específicos de las instancias individuales, pero tal comprensión no debe ser un límite a fin de salvar "la unidad distintiva del proceso social" $y$, por tanto, captar las relaciones que subsisten entre acción y estructura social. La relevancia de la transmisión cultural, realizada por los mismos ligámenes sociales, el conjunto de condiciones de orden tecnológico y la misma forma del proceso social, de aquello que MacIver llama social causation, deben introducirse en la consideración comprensiva de los factores que concurren para crear el cambio social.

Cuando MacIver distingue entre orden cultural, civilización y orden social alude a un "esquema inclusivo" (que en ausencia de otros términos distintivos, llama cultura, civilización, sociedad) que se presenta como un "conjunto coherente". Para MacIver existe una tendencia natural de la evolución social "a la integración de estos tres ámbitos". Cada uno de ellos presenta una específica "forma de cohesión". Aun cuando toda sociedad tienda naturalmente a integrar entre sí estos diferentes órdenes, MacIver observa cómo uno de los mayores problemas de una sociedad compleja como la nuestra es el interadjustement entre orden cultural y orden tecnológico.

El orden cultural interactúa con la acción social en cuanto que esta última está orientada por los "intereses": la dimensión cultural corresponde a un preciso tipo de "interés" del agente social. Comprende los modelos ideales, las "inter-adap- 
RIS

REVISTA INTERNACIONAL DE SOCIOLOGIA

№ 39, SEPTIEMBRE-DICIEMBRE, 2004

MANUEL HERRERA y PEDRO CASTÓN

taciones", las tendencias de tipo valorativo y orientadas a los valores junto a los objetivos que presiden las acciones, cómo éstas se revelan en las costumbres, en las tradiciones, en las creencias, en el arte, en las filosofías y cómo también se reflejan en los errores y en las formas de vivir de los individuos y de las comunidades (MacIver, 1964:288). Un cuadro, un poema, un drama, un film, un juego, una filosofia, una creencia religiosa, una catedral: todos son ejemplos de formas y modos en que "nos expresamos nosotros mismos". Y este "nosotros" es el nosotros que se constituye espontáneamente en una comunidad. Estas expresiones, dice MacIver, corresponden a una "necesidad interior" y no exterior: en tal sentido pertenecen al reino de la "cultura" y a la identidad de una comunidad y de un particular.

Aludiendo a autores como Marx, Sombart, Spengler, Mumford y sobre todo Alfred Weber, MacIver entiende por fenómeno auténticamente cultural la expresión de nuestra naturaleza en sus formas de vivir y de pensar, en nuestras relaciones cotidianas, en el arte, en la literatura, en la religión. En todo objeto que nos circunda se combinan tanto un elemento cultural, como un elemento ligado a la esfera de la civilización. Con una diferencia fundamental: mientras la civilización tiene un concreto estándar de medida, esto no sucede para las creaciones culturales, en las que siempre se refleja lo original de la personalidad humana, su aportación creativa y original insertada en una tradición. Por tanto, la distinción entre cultura y civilización también es resultado del proceso de diferenciación que se activa en las sociedades industriales avanzadas, mientras que en las sociedades simples dicha distinción no se ha desarrollado (MacIver, 1964:283).

El orden cultural es "el sistema de los intereses humanos primarios", mientras que el orden tecnológico o de civilización es el "aparato construido y utilizado para su consecución" (MacIver, 1964:285-286). La distinción entre cultura y civilización es útil y eficaz, ya que ayuda a comprender algunos de los problemas de la adaptación social y actúa "contra aquellas perspectivas que reducen los fenómenos culturales y los sistemas que los componen a meras derivaciones" (MacIver, 1964:288).

El orden cultural puede ser uniforme y homogéneo, como generalmente se presenta en las sociedades simples, pero también conflictivo y contradictorio (sociedades modernas y complejas) (MacIver, 1964:288). Respecto al "universalismo" del desarrollo tecnológico, los diferentes sistemas de valores siempre revelan de forma muy marcada sus rasgos distintivos, incluso cuando los diferentes sistemas culturales presentan diversos grados de cohesión, de inter-adaptación, que cambian considerablemente para grupos diferentes en el interior de las más grandes unidades sociales (MacIver, 1964:285).

Uno de los mayores problemas de las sociedades complejas es la inter-adaptación entre los órdenes anteriormente citados en cuanto que siempre interactúan y están sujetos a formas y ritmos de cambio diferentes (MacIver, 1964:287). Criticando la teoría de Ogburn sobre el "retroceso cultural", MacIver sostiene que presuponer con ingenuidad que el rol de la cultura es el de adaptarse a las 
nuevas condiciones tecnológicas, que la cultura es el factor derivado respecto al factor precipitante y creativo de la tecnología, significa eludir que exista para la cultura un "estándar común de eficacia", que, sin embargo, sólo puede existir para un sistema de medios como es el tecnológico (MacIver, 1964:288).

No existe actividad humana que no esté empapada por cualquier tecnología, que puede ser considerada como la adaptación de un principio, de un método a un objetivo particular. El orden tecnológico de MacIver comprende no sólo la tecnología de base, el sistema económico, también el sistema político: ambos forman conjuntamente un complejo institucional coordinado, cuyo carácter siempre es cambiante según las funciones regulativas realizadas por un sistema o por otro. Se trata de sistemas que, como todos aquellos que se incluyen en el order of civilization, están "regulados" pero no "construidos" por la vida social.

El orden tecnológico también es llamado "utilitarista", en el sentido de que corresponde a la esfera de la actividad y de la evolución social sujeta al criterio de lo útil y de lo eficiente. A diferencia de la cultura, dice MacIver, la civilización siempre tiende a avanzar: no sólo avanza, sino que siempre avanza no conociendo fracturas catastróficas en la continuidad social, como, sin embargo, sucede en la dinámica socio-cultural (MacIver, 1964:294). A diferencia del proceso de civilización, que avanza según un criterio cumulativo y universal, la cultura continuamente está sujeta a regresiones, avances, y siempre de forma no lineal. Su dinámica es tal que su pasado no asegura su futuro, incluso porque mientras que la civilización es trasmitida mediante esfuerzos racionales, la cultura sigue un principio de difusión muy diferente, que MacIver llama de "inclinación simpática".

Merton ha insistido en que MacIver diferencia la esfera de la cultura y la esfera de la civilización a partir del esquema medios-fines. La civilización es la que viene considerada por el hombre como puramente instrumental, como medio, mientras que la cultura comprende todo objeto, actividad, idea, que venga considerada como fin en sí mismo. La dificultad esencial de esta distinción es individuada por Merton en el hecho de que se base en diferencias relativas a la esfera de la motivación. Fines subjetivos y fines objetivos, punto de vista del actor y punto de vista del observador: he aquí el problema que, según Merton, corre el riesgo de no ser solucionado por MacIver, en el sentido de que diferentes motivos pueden ser fundamentales para la misma actividad social o cultural. De esta forma una clase de individuos solamente puede perseguir la ciencia como fin en sí misma, y hacer una típica actividad cultural, mientras que quien considera a la ciencia como puramente instrumental se introduciría en la esfera de la civilización. La conclusión de Merton es que "una serie de categorías tan flexible es inadecuada ya que los productos sociales tienden a tener el mismo significado social independientemente de la motivación que los produce" (Merton, 1936:110). Sin embargo, Merton ha reconocido un pleno valor analítico a las categorías de culture y civilization, tal y como han sido formuladas por la sociología de la cultura de Alfred Weber (Merton, 1936:111). 
RIS

REVISTA INTERNACIONAL DE SOCIOLOGta

NN 39, SEPTIEMBRE-DICIEMBRE, 2004

MANUEL HERRERA Y PEDRO CASTÓN

En su obra Social Causation MacIver responde a esta crítica subrayando cómo la distinción entre orden cultural y esfera de la civilización no coincide con la de medios y fines, y no contempla solamente motivaciones individuales, sino sistemas o modelos de actividades establecidas por grupos (MacIver, 1964: 285-286). Si los instrumentos de la tecnología de base son ideados en primer lugar para controlar las cosas, el complejo económico-político sirve para controlar las relaciones de los hombres. El complejo económico-social en tal sentido es un aspecto del orden social, el aspecto regulativo, pero no agota su vida más profunda. Mientras que las relaciones económicas y políticas simplemente son "regulativas", existe un ámbito de la realidad social, la vida humana y la red de relaciones que la cruzan, que respecto a estas esferas regulativas tiene su propia vitalidad y creatividad. Según MacIver, una de las razones por las que la ciencia sociológica se ha desarrollado puede buscarse en el creciente reconocimiento del orden social existente por derecho propio, en cuanto que comprende el esquema fundamental de las relaciones humanas.

La clasificación en sistemas, órdenes y esferas implica, de esa forma, el traslado a una base unitaria del orden social, que es la red de acciones singulares y de las relaciones a que dan vida. El sociólogo debe ligar la acción social y la "valoración dinámica" que la distingue con el conjunto de factores objetivos organizados selectivamente por ella (MacIver, 1964:297). Es decir, todo comportamiento consciente pone en relación medios y fines, pero el proceso que establece esta relación, para MacIver, "es contingente e incluye una atribución de causalidad que puede o no ser confirmada por la experiencia" (MacIver, 1964:298).

La valoración dinámica es el juicio que se traslada a la decisión de obrar y se diferencia del juicio post-mortem de la historia o de la ciencia social en que es doblemente contingente (MacIver, 1964:298). Según MacIver, las "contingencias" que gravan sobre la acción son tres: la primera se verifica en la estructura del sistema interno y es subjetiva, la segunda contempla la relación entre interno y externo (relación entre medios y fines), y la tercera alude a la dinámica del orden exterior. Preliminarmente la acción consciente se constituye como decisión entre alternativas; continuación de una decisión tomada en tal sentido es, posteriormente, la reorganización selectiva de algunos factores externos contemplados por el actor como medios. La valoración dinámica "implica un tipo de juicio causal que es diferente de la atribución post-factum de la causalidad típica de las ciencias sociales, en cuanto que es doblemente especulativa (...), la selectividad de la valoración dinámica, en cuanto que examina la situación antecedente a la decisión como fórmula de las alternativas de la acción, la somete a diferentes tipos de contingencia y de riesgo práctico" (MacIver, 1964:300); en fin, puede cambiar el objetivo dominante, puede ser equivocado el nexo medios-fines, el orden físico, que se presupone bajo control y puede revelar dificultades inesperadas.

De esta forma, el problema de la explicación de la acción social es el siguiente: existen factores de orden y realidades diferentes que entran y cooperan en el obrar 
que "de cualquier forma deben actuar conjuntamente, deben ser confrontables y coordinarse en cuanto que deben actuar con o contra el otro en la determinación del fenómeno" (MacIver, 1964:301). Por tanto, en la valoración dinámica todos los factores que determinan un comportamiento consciente son introducidos en un orden específico (MacIver, 1964:301-302).

Estudiar la social causation no significa limitarse a la acción social individual, sino que es necesario tratar de explicar cómo y por qué individuos diferentes actúan de forma diferente en situaciones diferentes: no es la cuestión de cómo nos comportamos tú o yo, dice MacIver, sino de cómo "nos comportamos". Es el "nosotros" de los seres asociados, cuyas formas de comportamiento, sean iguales, complementarias, diversas u opuestas, entre sí son afines y en cierto sentido interdependientes y básicas para el análisis de la causalidad social.

No solamente existe una "valoración dinámica" individual, sino que también existe una "valoración dinámica" del grupo, valoraciones que son "iguales y convergentes". A su vez, el grupo es un foco de valoración que impone su modelo sobre políticas, acontecimientos, opiniones y credos de sus miembros. No solamente existe un estilo, una forma de pensar, un modo de vivir, de obrar individual, sino que también existe con respecto al grupo (MacIver, 1964:304). En este sentido MacIver distingue tres tipos de fenómenos sociales:

1. Cambios en las costumbres, en los estilos, en los usos, en las tendencias de la opinión y en los hechos estadísticos generales (natalidad, crimen, suicidio, frecuencia de los matrimonios, etc.) que no expresan la actividad concordada o colectiva del grupo.

2. Fenómenos (políticos, movimientos sociales organizados, revoluciones o demostraciones, acuerdos sociales de todo tipo) en los que los individuos están más o menos de acuerdo en la valoración de la situación y activan una acción concordada.

3. Productos de una "coyuntura social": es un tipo de fenómenos sociales que incluye, según MacIver, las más grandes estructuras del orden social, el nivel de división del trabajo, las formas del control político-económico, las diversas formas de desorganización social. Fenómenos que pueden depender de un proyecto inicial o no.

¿Cuál es la relación entre valoración dinámica individual y valoración dinámica de grupo? MacIver pone el ejemplo del descenso de la tasa de natalidad que se ha producido en los últimos setenta años en los países occidentales. Evidentemente, no es el resultado que emerge del acuerdo de una serie de núcleos familiares que deciden romper con el comportamiento de las generaciones precedentes. Lo que ha sucedido es que han cambiado las condiciones objetivas en que vivía la gente: progresivamente ha aumentado el porcentaje de ocupados en el sector industrial e igualmente han aumentado los costes para el mantenimiento de una familia (MacIver, 1964:310). 
RIS

REVISTA INTERNACIONAL DE SOCIOLOGIA

NN 39, SEPTTEMBRE-DICIEMBRE, 2004

MANUEL HERRERA y PEDRO CASTÓN

Entre valoración dinámica individual y de grupo existe una auténtica interdependencia, y no uni-direccionalidad. Regresando al ejemplo anterior, es evidente, dice MacIver, que el complejo cultural que sostenía el principio de la natalidad ha sido minado por cambios tecnológicos y económicos, y que el valor social ligado a una alta tasa de natalidad fue contingente a la coexistencia de una alta tasa de mortalidad. Todo un conjunto de cambios objetivos (urbanización, división del trabajo, especialización, disminución de la tasa de mortalidad infantil, costes crecientes del mantenimiento de la familia, etc.) ha sido la base para el aumento de una creciente tensión y no armonía entre valoraciones dinámicas individuales y complejo cultural (1964:313). El resultado ha sido una "aceptación social implícita", al menos por parte de los mismos grupos, de la familia reducida.

Más allá de esta interacción entre esquemas de valoración, para MacIver existe el fenómeno que denomina "coyuntura social", por el que una combinación y oposición de elementos dinámicos puede producir estructuras de notable complejidad que son el resultado de adaptaciones funcionales complejas. En este caso las acciones intencionales de los hombres, que se expresan en una diversidad de valoraciones dinámicas, llevan a "resultados imprevistos". La estructura más grande de toda sociedad, su "complejo institucional", es un resultado de este tipo (MacIver, 1964:314).

La sociedad está construida por una multitud de actividades que forman y reforman sus asociaciones, establecen relaciones cambiantes de un tipo o del otro, hasta que se constituye un modelo general que no es fruto de algún plan preestablecido. Aunque tenga éxito, un espíritu revolucionario que trate de controlar o rehacer una estructura, dice MacIver, construirá una realidad diferente de la proyectada. El error de fondo, por ejemplo de Malthus, incluso de Darwin y de las sociologías de Gumplowicz, Lapouge o Spencer, es el de la atribución de resultados totales a una entidad o causa particular. La sociología y sus métodos, por el contrario, deben hacer propia la convicción de que el problema real siempre es la forma en que diversos factores están en relación en los procesos de cambio social. Todo factor tiene un rol, pero siempre en una compleja interdependencia con las cosas. Aunque las unidades que emergen sean imperfectas, en un continuo proceso de formación y de disgregación, existe una tendencia a una especie de integración (MacIver, 1964:321). De esta forma, la organización social presenta una y otra vez una particular conformación congenial tanto respecto al sistema de los fines fijado en la esfera cultural, como al sistema de los medios definido en la esfera tecnológica, pero la relación entre estas esferas siempre depende de una "valoración dinámica" que es la que activa una ponderación de los factores y lo hace no según escalas objetivas sino que "varía con todo estado de ánimo y de situación".

En conclusión, en toda acción social emerge una nueva relación dinámica entre los siguientes aspectos de sistemas que han experimentado en el tiempo una continua readaptación: 
1. Una serie de fines (incluidos los impulsos conscientes de todo tipo) que emergen de un conjunto cultural concreto y que encuentran particular expresión en el proceso de valoración dinámica.

2. Una serie de técnicas o controles derivados del aparato de una particular civilización y aplicados a fines específicos.

3. Una serie de relaciones sociales que se introducen en un más amplio sistema social, organizado conforme a los fines particulares y al mismo tiempo constituye un aspecto de uno o ambos de los puntos 2 y 3 , como entidad si no como objetivo de la acción.

4. Una serie de condiciones biofísicas relativas a los pre-requisitos de la acción particular.

\section{ACCIÓN SOCIAL Y SISTEMA POLÍTICO: LA COMUNIDAD Y EL ESTADO}

Como ya se ha señalado, el otro gran tema que caracteriza el itinerario científico e intelectual de MacIver está constituido por el intento de elaborar las líneas esenciales de una sociología y filosofia política. Un breve examen de este intento nos permitirá completar el análisis de su teoría de la acción social.

La sociología política de MacIver, cuya tesis central es que "el Estado no tiene ninguna finalidad", concretamente se desarrolla en The Modern State (1926) y en The Web Government (1947). El Estado en sí mismo no puede tener y expresar fines propios, tan sólo es un medio, un instrumento al servicio de los fines de la persona y de las comunidades donde se realiza un aspecto fundamental de su ser: su sociabilidad. No está de más subrayar la particular actualidad de esta intuición y el hecho, como han mostrado varios críticos de la obra de MacIver, de su intersección con una visión típicamente "federalista" de la relación entre Estado y sociedad. Una intuición que, por una parte, tiene su fundamento en una precisa concepción sociológica, pero, por otra, en la profunda convicción filosófica de que los fundamentos del orden político siempre son, al final, el respeto y la justicia.

La sociología de la acción socio-política reclama y presupone en MacIver un consciente ligamen con la antropología filosófica: según este ligamen, el hombre es, aristotélicamente, un animal "sapiente", en primer lugar un homo sapiens, y no como desean el conductismo o el pragmatismo contemporáneos un homo faber.

$\mathrm{Ni}$ la sociología política, ni una teoría del Estado pueden renunciar a la filosofía política: gobernar significa, en primer lugar, resolver el problema de establecer los fines (MacIver, 1947:413). MacIver expresa esto afirmando que la sociedad humana y política siempre está guiada por "mitos". Estos son concebidos como "aquellas ideas y opiniones, cargadas de juicios de valor, que tienen los hombres, y conforme a las que viven" (MacIver, 1947:8). Según MacIver, las mismas relaciones sociales, "verdadero tejido de la sociedad humana", están "generadas 
y alimentadas por mitos". La historia del hombre no es comprensible sin una referencia cultural: toda civilidad, toda era, toda nación, posee su complejo de mitos característico (MacIver, 1947:9).

Aunque pueda existir interdependencia entre el ámbito del mito (orden cultural) y el ámbito general de la técnica (orden de la civilización), esta segunda esfera no puede sustituir a la primera (MacIver, 1964:11). El análisis del rol que el "mito" tiene en el surgir de la acción política también influencia la interpretación de MacIver del origen del gobierno y del poder. Rechaza en primer lugar la teoría hobbesiana. En ella encuentra una verdad parcial que genera demasiados errores (MacIver, 1964:11). La fuerza privada de autoridad no está en situación de crear orden y la explicación que avanza MacIver es de naturaleza tanto sociológica, como filosófica. La misma autoridad es una función de la cohesión social, de la integración, del sentido de la comunidad: dura en la medida en que depende de los mitos dominantes de aquellos sobre los que se ejerce, mitos que derivan, añade, de la naturaleza social del hombre y que a su vez la influencian.

Al mismo tiempo MacIver revela los límites de las teorías contractualistas: aludiendo a Burke y su idea de una sociedad "que existe no sólo entre los vivientes, sino entre los vivientes, los muertos y los por nacer", es decir, la idea de una profunda solidaridad moral que liga a las generaciones, revela cómo el concepto de contrato es muy limitado para comprender la naturaleza y los orígenes del Estado. El nacimiento de la autoridad y su permanencia están demasiado conectados al proceso de "institucionalización de un mito central". En el caso de una sociedad compleja como la que se desarrolla en los siglos de la historia occidental moderna, en la que a la homogeneidad cultural de los tipos precedentes de sociedad, en que existía una única religión, una única medida de los valores, una única enseñanza común, le sustituye la presencia de múltiples grupos con múltiples mitos, se llega al reforzamiento del único "mito" en situación de conciliar los diferentes mitos en conflicto: este mito central es para MacIver el mito de la democracia. Ésta se encuentra en situación de conciliar las "diferentes fidelidades contratadas" del ser social.

Según MacIver, en la época contemporánea, autoridad y orden sólo pueden subsistir si "se permite convivir a las diferentes fidelidades del hombre, adaptándose mutuamente en el ámbito de un ordenamiento único" (MacIver, 1964:11). Para alcanzar esta capacidad democrática es necesario un desarrollo gradual y lento: se trata de una forma de gobierno que jamás estará completamente actualizada y activada (MacIver, 1964:181). Una verdadera democracia no puede aceptar la identificación de Estado y comunidad, ni aquella entre Estado y nación. Vivimos, dice MacIver, en comunidades y no en Estados, que no poseen el carácter funcional de la comunidad. Es la comunidad la que emerge de la vida social, no el Estado (MacIver, 1964:199-200). La teoría de la democracia de MacIver, en virtud del análisis sociológico en el que se fundamenta, está orientada contra lo que denomina "omnipotencia de la soberanía": un tema que al día de hoy es central en el debate socio-político, y sobre el que MacIver se había anticipado. 


\section{CONCLUSIONES}

La sociología "comprensiva" de MacIver ha construido una teoría de la acción social a partir de la clasificación de los intereses sociales, de la distinción entre comunidad y sociedad, del concepto evolución social, y de la intuición de una sustancial armonía de la relación entre sociedad e individualidad. En el centro de esta teoría de la acción está la categoría de la "valoración dinámica" que, al menos analíticamente, ubica al actor social como la única unidad de toda dinámica sociológica. Con la distinción entre orden cultural, tecnológico y social, MacIver ha tratado de evitar que la teoría sociológica identifique en la estructura social el único objeto de estudio. En este sentido, ha intentado construir una teoría de la acción social orientada, por lo menos en parte, a aquel paradigma denominado "individualismo metodológico".

Por otra parte, como ha recordado Alpert (1964:288), para MacIver la sociología no puede ser una "ciencia autárquica": debe integrar sus resultados tanto con la reflexión filosófica, como con la historia y la ciencia política. Según nuestro autor, la comprensión de la sociedad comienza por nuestra personal experiencia como miembros de la misma sociedad y no se agota jamás debido a la complejidad de los mismos fenómenos sociales.

En un terreno de caos literario e intelectual, MacIver ha aportado claridad de pensamiento y facilidad expositiva, dejando una gran cantidad de obras que incluso hoy nos interrogan y en las que se han formado estudiosos como D. Bell, C.H. Page, R.K. Merton y R. Nisbet.

La teoría de la acción social de MacIver encuentra una fértil prolongación y una coherente aplicación en su esfuerzo por definir una apropiada teoría democrática en la que "la teoría del Estado es la teoría de una asociación sin una unidad más inclusiva, una teoría que busca medios y no fines", y en la que la teoría social es de tal forma que "para comprender cómo es el Estado y cómo cambia, en primer lugar es necesario afianzar el significado de la comunidad y de las estructuras y procesos de la sociedad" (Spizt, 1964:295).

En efecto, en MacIver, la importancia atribuida al actor social, a su capacidad de construir una red de relaciones sociales alrededor de normas compartidas, se conjuga con una ética del reconocimiento del otro, igualmente encaminada a fundar un sistema social pluralista junto al derecho de toda comunidad y cultura a defender la propia identidad. Para MacIver, como se ha observado, "la cultura es distinta, única; pero es única como estilo ya que es la forma con la que un grupo o pueblo aprende a expresarse a sí mismo en lo que le es característico - y el estilo continuamente cambia -" (MacIver, 1964:283). En este esfuerzo de MacIver se inserta el intento de recuperar la dialéctica Kultur/Zivilisation, del sentido de la "especificidad" de toda comunidad, de la prioridad de la nación sobre el Estado. Pero también una concepción federalista que no sólo representa el desarrollo más natural de toda auténtica democracia, sino la única respuesta posible a la 
multi-group society. Una concepción que, aunque conectada a su teoría social, merecería una profundización que en el contexto del presente estudio no se ha podido llevar a cabo.

\section{REFERENCIAS BIBLIOGRÁFICAS}

ALLODI, L. (1988), "Presupposti tönniesiani al pensiero di Alfred Weber”, Annali di Sociología, II, pp. 169-178.

(1991), Alfred Weber. Una introduzione, Roma, Armando Editore.

(1997), "La sociología dopo la filosofia. Un confronto fra H. Arendt e K. Mannheim", Rassegna Italiana di Sociologia, IV, pp. 601-627.

(1998) “Mutamento sociale e metodo causale-significativo nell'opera di R.M. Maclver", Rassegna Italina di Sociología, V, pp. 234-257.

ALPERT, H. (comp.) (1953), Robert M. Maclver: teacher and sociologist, Northampton, Metcalf Publ.

(1964), "Robert M. Maclver's Contributions To Sociological Theory", en M. Berger, T. Abel y C.H. Page (comps.) (1964), pp. 286-292.

(1972), “Maclver Robert M.”, International Encyclopedia of The Social Sciences, D.L. Sills (Editor), Nueva York, The Mac Millan Publishers, pp. 513-515.

BERGER, M., T. ABEL y C.H. PAGE (comps.) (1964), Freedom and Control in Modern Society. In Honor of R. M. Maclver, Nueva York, Octagon Books.

BOUDON R. (1969), Les méthodes en sociologie, Presses Universitaires de France, París.

(1979), Logique du social, París, Librairie Hachette.

(1977), Effets pervers et ordre social, París, Presses Universitaires de France.

(1973), Le place du désordre. Critique des théories du changement social, Presses Universitaires de France, París.

(1989a), "Razionalità soggettiva a disposición”, en Sciolla L., Ricolfi L., (comps.) (1989).

(1989b), “Razionalità e sociologia”, en Sciolla L., Ricolfi, L. (comps.), (1989).

(1995), Le juste et le vrai, Etudes sur le l'objectivité des valeurs et de connaissance, París, Fayard.

(1997), "L'individualismo metodologico e le tradizioni sociologiche", en Petrini-Viale (1997).

BOUDON R. y F. BOURRICAUD (1982), Dictionnaire critique de la sociologie, Presses Universitaires de France, París. 
GINER, S. (1971), La sociedad masa: Sociologia y Conflicto social, Seminarios y Ediciones, Madrid.

(1974), El progreso de la conciencia sociológica, Península, Barcelona.

(1979a), Sociedad masa: crítica del pensamiento conservador, Península, Barcelona.

(1979b), "Prólogo: Ferdinal tönnies y la ciencia social moderna", en F. Tönnies, Comunidady Asociación, Península, Barcelona.

KLUCKHOHN, C. y A.L. KROEBER (1952), Culture: a Critical Review of Concepts and Definitions, Nueva York, Vintage Books.

MacIVER, R.M. (1926), The Modern State, Oxford-New York, Oxford University Press.

(1928), Community. A sociological Study, Londres, Mac Millan.

(1932), "Interests", Encyclopaedia of Social Sciences, vol. 8, Nueva York, Macmillan, pp. 144-48.

(1933), Society. Its Structure and Changes, Nueva York, Ray Long and R. Smith.

(1936), "The Historical Pattern of Social Change", Journal of Social Philosophy, 2, pp. 35-54.

(1931), "Is Sociology a Natural Science?", Publications of The American Sociological Society, Fox, Nueva York, pp. 25-35.

(1947), The Web of Government, Nueva York, Macmillan.

(1955), Academic Freedom in Our Time, Columbia University Press, Nueva York.

(1964), Social Causation (1942), Nueva York, Harper Torchbooks.

(1968), As A Tale That Is Told. The Autobiography of R.M. MacIver, Chicago, The University of Chicago Press.

MERTON, R.K. (1936), "Civilization and Culture", Sociology and Social Research, NovemberDecember, pp. 103-113.

(1970), Science, Tecnology and Society in Seventeenth Century England, Nueva York, Harper and Row.

(1994), Propedeutica sociologica, Monduzzi Editore, Bolonia.

NAGEL, E. (1956), "Social Causation", Logic Without Metaphysics, The Free Press, Glencoe.

(1961), The Structure of Science, Nueva York, Harcourt, Brace and World.

NOLTE E. (1993), Intervista sulla questione tedesca ieri e oggi, (comp. A. Krali), Laterza, Bari.

PETRINI, A.M. y R. VIALE (comps.) (1997), Individuale e collettivo. Decisione e razionalità, R. Milán, Cortina Editore. 
RIS

REVISTA INTERNACIONAL DE SOCIOLOGIA

№ 39, SEPTIEMBRE-DICIEMBRE, 2004

MANUEL HERRERA y PEDRO CASTÓN

POGGI, G.F. (1962), Teoria politica e sociologia in Maclver, Milán, Franco Angeli.

ROSSI, P. (1957), “Cultura e Civiltà come modelli descrittivi”, Rivista di Filosofia, XLVIII, pp. 274-297.

(1975a), Introduzione, en Rossi-Mori-Trinchero (1975).

(1975b), L'impostazione weberiana e il nesso tra causalità e posibilità, en Rossi-Mori-Trinchero.

ROSSI, P., M. MORI y M. TRINCHERO (comps.) (1975), Il problema della spiegazione sociologica, Loescher, Turín.

SCIOLLA, L. y L. RICOLFI (comps.) (1989), Il soggetto dell'azione. Paradigmi sociologici ed immagini dell'attore sociale, Milán, F. Angeli.

SCIOLLA, L. (1989), "Razionalità, intenzionalità e analisi della mente", en Sciolla L., Ricolfi L. (comps.)

SOROKIN, P. (1941), Social and Cultural Dynamics, vol. IV, Nueva York, 1937-41.

(1947), Society, Culture and Personality, Nueva York.

(1956), Fads and Foibles in Mode Sociology and Related Sciences, H. Regnery Company, Chicago.

SOMBART, W. (1911), "Technik”, Archiv für Sozialwissenschaft und Sozialpolitik.

SPITZ, D. (1964), “Robert M. Maclver's Contributions To Political Theory”, en Berger M., Abel T., Page C.H. (1964)

SPITZ, D. (comp.) (1969), Politics and society by Robert M. Maclver, Atherton Press, Nueva York, con una amplia bibliografia dedicada a Maclver (pp. 533-543).

TÖNNIES, F. (1887), Gemeinschaft und Gesellschaft, Leipzieg, O.R. Reislad (edición española, Comunidad y Asociación, Península, Barcelona, 1979).

TENBRUCK, F. (1985), “I compiti della sociologia della cultura”, Annali di Sociologia, 1, pp. 71-95.

WEBER, A. (1927), Ideen zur Staats und Kultursoziologie, Braun, Karlsruhe.

(1931), “Kultursoziologie”, en A. Vierkandt y F. Enke (comp.), Handwörterbuch der Soziologie, Stuttgart Verlag.

(1950), Kulturgeschichte als Kultursoziologie, Munich, Piper.

(1951), Prinzipien der Geschichts und Kultursoziologie, Munich, Piper. 